\begin{abstract}
The Tangram Help/Hurt Task is a laboratory-based measure designed to simultaneously assess helpful and hurtful behavior. Across five studies we provide evidence that further establishes the convergent and discriminant validity of the Tangram Help/Hurt Task. Cross-sectional and metaanalytic evidence finds consistently significant associations between helpful and hurtful scores on the Tangram Task and prosocial and aggressive personality traits. Experimental evidence reveals that situational primes known to induce aggressive and prosocial behavior significantly influence helpful and hurtful scores on the Tangram Help/Hurt Task. Additionally, motivation items in all studies indicate that tangram choices are indeed associated with intent of helping and hurting. We discuss the advantages and limitations of the Tangram Help/Hurt Task relative to established measures of helpful and hurtful behavior.
\end{abstract}

Keywords: Helpful, Hurtful, Prosocial, Aggressive, Validation

\title{
Helping and Hurting Others: Person and Situation Effects on Aggressive and Prosocial Behavior as Assessed by the Tangram Task
}

Although prosocial and aggressive behavior are conceptually distinct, they often are inversely related-especially in short-term real-world contexts. When people engage in hurtful behavior toward a target person, they seldom simultaneously engage in helpful behavior toward that same target. Of course, specific types of aggressive behavior (e.g., instrumental aggression) may involve harming another with an overarching prosocial goal. Therefore, it is important to assess aggressive and prosocial behavior simultaneously. To this end, the current studies were conducted to further test the convergent and discriminant validity of the recently developed Tangram Help/Hurt Task (Saleem, Anderson, \& Barlett, 2015). Additionally, these studies 
replicate some findings from prior studies, an important task given recent failures to replicate scientific findings (e.g., Pashler \& Wagenmakers, 2012; Spellman, 2013).

Myriad domain-specific theories provide excellent explanations of aggression and altruism, but broader theories better integrate the many possible variables that link helpful and hurtful behavior. Thus, we employ the General Aggression Model (GAM; e.g., Anderson \& Bushman, 2002; DeWall, \& Anderson, 2011; Warburton \& Anderson, in press) and its broader derivative, the General Learning Model (GLM; e.g., Barlett \& Anderson, 2013) as organizing frameworks for this research. Both GAM and GLM are dynamic integrative social-cognitive learning-based theories relevant to prosocial and aggressive behavior. Across three correlational and two experimental studies, we test specific hypotheses about person (i.e., cross-sectional individual differences) and situation (i.e., experimental manipulations) variables that theoretically (and in many cases, empirically) should relate to the Tangram Help/Hurt Task.

\section{GENERAL LEARNING AND AGGRESSION MODELS}

Both GAM and GLM can be partitioned into two inter-related sets of processes:

proximate and distal. The proximate versions posit that a person's behavior is influenced by two types of input variables: the person and the situation. Person variables consist of genetics, traits, current states, beliefs, attitudes, values, scripts, and other variables that constitute one's relatively enduring characteristics. Situation variables are features of the environment that influence an individual's thoughts, feelings, arousal, and actions (e.g., rewards, cues, pain, frustration). These input factors influence a person's present internal state, which consists of cognition, affect, and arousal. These internal states influence appraisal and decision processes that precede behavior. Whether the ensuing behavior is thoughtful or impulsive is based on the input variables, changes to the internal state, the immediate appraisal, and (when possible) reappraisal. 
Support for both models is well established (Anderson \& Bushman, 2002; Gilbert, Daffern, \& Anderson, in press; Prot et al., 2015). For example, personality traits such as narcissism, impulsivity, and neuroticism are associated with aggressive cognitions and feelings, which, in turn, increase the likelihood of aggressive behavior. Similarly, prosocial personality traits (e.g., empathy, perspective taking, and forgiveness) are associated with prosocial cognitions and feelings, which increase the likelihood of prosocial behavior (e.g., Davis, 2015). A wide range of situation factors (e.g., uncomfortable temperature, provocation, violent media, presence of a gun) can increase aggressive behavior by changing internal state variables. Prosocial situational cues such as the degree of similarity between the observer and the victim, religious primes, and prosocial media are associated with prosocial behavior.

\section{The relation between aggressive and prosocial behavior.}

Conceptually, aggressive and prosocial behavior appear to be opposites, one involving behavior intended to harm another person, the other involving behavior intended to help another person. Theoretically, they share the very important feature of being largely defined by intent rather than actual outcome (e.g., Anderson \& Bushman, 2002; Batson, 2014). For this reason, psychological processes underlying both types of behavior may be similar, involving both fairly automatic (impulsive) perception-decision-action sequences as well as more resource-intensive controlled sequences. Indeed, Hirsh, Galinsky and Zhong (2011) suggest that the common factor behind seemingly contradictory prosocial and antisocial outcomes is a disinhibited state characterized by reduced response conflict. Such disinhibition influences the most salient response in a given context based on either trait tendencies or strong social cues. Similarly, Graziano and Habashi (2010) provide an excellent theoretical integration of the similar processes underlying prejudice (which is strongly aligned to outgroup aggression) and prosocial behavior. 
Despite these theoretical insights, most existing measures assess either aggressive or prosocial behavior (Bushman \& Anderson, 1998; MaCrae \& Johnston, 1998; Piff et al., 2010; van Baaren, Holland, Dawakami, \& van Knippenberg, 2004). This is problematic as researchers cannot equate a lack of aggressive (or prosocial) behavior using any of these tasks as a prosocial (or aggressive) response ${ }^{1}$. The Tangram Help/Hurt Task (THHT) simultaneously assesses helping and hurting behavior while allowing a third non-aggressive non-prosocial option (see Saleem et al., 2015 for a detailed description). In six studies that article provided initial convergent and discriminant validity evidence for the THHT. Correlational evidence revealed that THHT hurting scores positively correlated with aggressive personality constructs, i.e., trait aggression, narcissism, control aggression schema, state hostility, and sensation seeking. THHT helping scores positively correlated with prosocial personality constructs , i.e., trait prosocialness, empathy, perspective taking, and agreeableness. Also, experimentally manipulated social contexts known to influence aggressive and prosocial behavior (i.e., provocation, empathy) significantly affected THHT hurting and helping scores. In addition, selection of hard puzzles was significantly predicted by intentions of harming the other participant, whereas, selection of easy puzzles was significantly predicted by intentions to help the other participant. Finally, responses on the THHT were not associated with perception of task difficulty.

The current studies add to this area in at least two ways. First, they tested previously unexplored, theoretically relevant personal and situation effects on THHT performance. Second, the studies attempted to replicate some key findings of our initial THHT studies, an especially important goal in light of recent attention to (lack of) replication in psychological science.

\section{STUDIES 1-3: CROSS-SECTIONAL TESTS OF THE HELP/HURT TANGRAM TASK}

\footnotetext{
${ }^{1}$ A notable exception is the Help/Hurt button (Liebert \& Baron, 1972). However, this measure has been validated only with children in lab settings, the primary measure is the length of time the Help or Hurt button is pressed and not the decision to choose helping or hurting behavior, and lacks a third non-aggressive, non-prosocial option.
} 
Studies 1-3 tested THHT convergent and discriminant validity using a correlation design. We aimed to replicate the relations between THHT scores and trait aggression, narcissism, state hostility, empathy, and perspective taking found in Saleem et al. (2015). We also examined correlations between THHT scores and person constructs not previously tested. Specifically, Study 1 tested correlations between tangram helping and hurting choices and trait forgiveness, state hostility, and value importance of power and benevolence. Study 2 tested correlations between trait empathy, perspective taking, narcissism, social desirability, and tangram choices. Study 3 tested correlations between tangram choices, social responsibility, morality, and social desirability. Motivations for tangram choices were assessed in all three studies.

\section{Methods}

\section{Participants}

Study 1. Students from a Midwestern University (111 female; 122 male) participated in the current study for course credit. The mean age was $19.58(S D=1.77)$ years.

Study 2. Amazon Mturk workers (N=258) participated for monetary compensation. Fifteen participants were dropped for technical problems. Two indicated they did not understand the THHT after watching the online instructional video. Of the remaining 241 participants, 124 were female, 117 were male, Mage $=36.10$ years, $S D=12.21$.

Study 3. Amazon Mturk workers (N=239) participated for monetary compensation. Nine were dropped for technical problems. Of the remaining 230 participants, 89 were female and 139 were male, Mage $=34.77$ years; $S D=11.64$.

\section{Materials}

Table 1 lists the descriptive statistics for all measures used in Studies 1-3. It also shows which measures were included in each study. 
Tangram Help/Hurt Task. The tangram assignment table was used by participants to assign 11 tangrams to the "other participant", and was supposedly used by the "other participant" to assign 11 puzzles for the participant to complete within the ten minute time limit (see Saleem et al., 2015 for a detailed description of this task).

Tangram assignment motivation. Participants indicated their agreement with two ${ }^{2}$ statements assessing their motivation to help (e.g., I wanted to help the other participant win the prize) and two assessing motivation to hurt (e.g., I wanted to make it difficult for the other participant to win the prize) on a 1 (strongly disagree) to 5 (strongly agree) scale.

\section{Measures Assessing Convergent Validity}

Trait aggression, trait narcissism, state hostility, and importance of power have been associated with aggressive behavior in previous literature (e.g., Anderson \& Bushman, 2002; Joireman et al., 2003; Tremblay \& Ewart, 2005). Similarly, trait forgiveness, empathy, perspective taking, moral reasoning, social responsibility, and importance of benevolent values have been associated with prosocial behavior (e.g., Dovidio et al., 2006; Penner et al., 1995; Schwartz, 2010). Thus, we expected these trait measures to correlate with THHT.

Trait aggression. We used the 29-item Buss-Perry Aggression Questionnaire (BPAQ: Buss \& Perry, 1992) in Study 1, and the 12-item brief measure (Webster et al., 2014) in Study 2. Participants indicated agreement with statements (e.g., "If somebody hits me, I hit back") on a 1 (Extremely uncharacteristic of me) to 5 (Extremely characteristic of me) scale.

Trait empathy and perspective taking. Trait empathy and perspective taking were assessed using the Interpersonal Reactivity Index (Davis, 1983). Participants indicated their agreement with statements on a 1 (Does not describe me well) to 5 (Describes me very well)

\footnotetext{
${ }^{2}$ Due to programming errors, motivation to help in Study 1 and Study 4 was assessed using a single item (I wanted to help the other participant win the prize).
} 
rating-scale. Examples of perspective-taking and empathy items include "I sometimes try to understand my friends better by imagining how things look from their perspective," and "I often have tender, concerned feelings for people less fortunate than me."

Trait forgiveness. The 10-item Trait Forgiveness Scale (Berry, Worthington, O'Connor, Parrott, \& Wade, 2005) was used to assess forgiveness. Participants indicated their agreement with statements (e.g., "I am a forgiving person") on a 1 (strongly disagree) to 5 (strongly agree) scale. Two items were not displayed properly due to programming errors (I feel bitter about many of my relationships; There are some things for which I could never forgive even a loved one). These two items were removed and the remaining 8-item scale was averaged together.

Prosocial Inventory. Penner's (1995) prosocial inventory was used to assess social responsibility (15-items) and moral reasoning (8-items). Participants indicated their agreement with statements on a 1 (strongly disagree) to 5 (strongly agree) scale. Examples of social responsibility and moral reasoning include "If a good friend of mine wanted to injure an enemy of theirs, it would be my duty to try to stop them" and "My decisions are usually based on my concern for other people". Subscales assessing empathy, perspective taking, and prosocial behavior were not included because previous studies have established the relationships between these constructs and tangram choices (Saleem et al., 2015).

Narcissism. Participants read 16 pairs of statements and selected the option that represents them within each pair (e.g., "I like to be the center of attention") (Ames, Rose, \& Anderson, 2006).

State hostility. We used a shortened version of the State Hostility Scale (Anderson, Deuser, \& DeNeve, 1995). Participants indicated the extent to which they currently feel various 
antisocial (12-items) and prosocial (10-items) emotions (e.g., "I feel furious", "I feel happy") using a 1 (Strongly Disagree) to 5 (Strongly Agree) scale.

Schwartz Value Questionnaire. Participants rated the relative importance of value statements according to their salience as guiding principles in their life using a 9-point scale $(1=$ against my values, $9=$ extremely important). Only items from the benevolence and power subscales were included because previous work suggests that these are most directly related to aggressive and prosocial behavior (Schwartz, 2010; Tremblay \& Ewart, 2005).

\section{Measures Assessing Discriminant Validity}

Previous studies indicate that tangram choices are not influenced by participants' perception of Tangram Task difficulty, achievement motivation, or emotion regulation (Saleem et al., 2015). The present study tests if individual differences in social desirability concerns influence tangram choices.

Social Desirability. Participants indicated whether each of the 11-statements (e.g., I'm always willing to admit it when I make a mistake) were true/false in describing them (Reynolds, 1982). Socially desirable responses were coded as 1 and other responses were coded as 0 , all 11 statements were summed together.

\section{Overall Procedure}

Participants completed an informed consent and were told that they would be completing a puzzle task with another participant. Participants received standardized tangram instructions in person in Study 1 and online through a video in Studies 2 and 3. They practiced solving tangrams with a practice packet in Study 1 and saw examples of tangrams being solved through a video in Studies 2 and 3. They then answered trait measures specific to each study (Table 1). Next, they assigned 11 tangrams from the tangram assignment table to the other participant. Then, they 
completed questions assessing motivation for tangram assignment and demographic information.

Next, participants answered open-ended questions designed to assess suspicion ${ }^{3}$. Finally, participants were debriefed, thanked, and dismissed.

\section{Results}

\section{Preliminary analyses}

As in previous studies, two scoring methods for the THHT were used: (a) separate helping and hurting scores derived from summing the number of easy puzzles and hard puzzles and subtracting 1 from these scores ${ }^{4}$, and (b) an overall tangram difference score obtained by subtracting the hurting score from the helping score (see Saleem et al., 2015 for a discussion regarding the different scoring methods). We report results using both scoring techniques.

\section{Studies 1 to 3 General Findings}

Table 2 reports zero-order correlations for all measures. Five general findings emerge. First, the helping, hurting, and the overall tangram scores were (necessarily) highly correlated. Second, participant sex was unrelated to tangram scores. This obviates the need to control for sex in more substantive analyses. Third, motivations to help and hurt the "other participant" strongly and consistently correlated with Tangram scores in exactly the expected pattern. Fourth, among the trait aggression subscales, the Tangram scores were consistently associated with physical aggression, anger, and hostility, and were least associated with verbal aggression. Finally, the Tangram scores correlated with other individual difference variables with which they should

\footnotetext{
${ }^{3}$ Participants were asked open-ended questions at the end of the survey such as "What did you think about the study?" and "Do you have any thoughts about the other person at this point?" As in previous studies (Saleem et al., 2015), participants who reported doubt about the presence of another participant were excluded from main analyses. The number of participants who were suspicious in Studies 1, 2, and 3, were Ns = 2, 11, 7, respectively.

${ }^{4}$ Because the task includes 10 puzzles per difficulty level and required 11 choices, participants have to pick from at least two categories. It is possible for someone to pick 10 medium tangrams and 1 easy (or hard) tangram to complete the 11 required. However, this individual is not necessarily intending to help (or harm) the other participant, because the other participant needed to complete only 10 tangrams to win the gift certificate. Thus, "helping" was operationally defined as the number of easy puzzles greater than 1. Similarly, "hurting" was defined as the number of hard puzzles greater than 1
} 
correlate (convergent validity) and yielded weak and/or essentially zero correlations with social desirability concerns, thereby providing evidence for discriminant validity.

The relations between the THHT and trait aggression, state hostility, empathy, perspective taking, narcissism, sex, and motivations to help/hurt replicated previous studies (Saleem et al., 2015). The relations between the THHT and forgiveness, values of power and benevolence, social responsibility, and moral reasoning provided new and theoretically confirming evidence of THHT validity.

\section{Meta-analysis of correlations.}

Six correlational studies - three in the present article and three in Saleem et al., 2015tested the relation between theoretically relevant individual difference variables and THHT performance. Several individual difference variables were assessed in more than one study. Thus, we meta-analyzed the correlations between the individual difference variables and THHT scores, employing the varying-coefficient model (Bonett, 2009; Krizan, 2010). As displayed in Table 3, all three THHT scores were significantly associated with the Buss-Perry composite score its four subscales, prosocialness, empathy, perspective taking, narcissism, state hostility, motivation to help, and motivation to hurt $(p s<.05)$. However, participant sex and social desirability did not significantly correlate with all three THHT scores. The average effect sizes should prove useful for power analyses when planning new research with the THHT.

\section{STUDY 4: SPIRIT-OF-GOD PRIME EFFECT ON THE TANGRAM TASK}

Study 4 tested the effects of a previously validated prime manipulation (Spirit-of-God) that is known to influence prosocial behavior, on THHT performance (Johnson et al., 2013). If the THHT validly assesses helping behavior, the Spirit-of-God experimental manipulation should increase helpful choices. Trait aggression and trait helpfulness were assessed pre- 
experimentally. We measured state hostility post-experimentally to see whether the effect of the prime on tangram choices was influenced by participant mood.

\section{Participants}

\section{Method}

One hundred forty-nine participants from a large Midwestern University completed the study for course credit. Seventeen were rated as suspicious based on their answers to a structured funnel debriefing; their data were deleted ${ }^{5}$. Of the remaining participants, 48 were male, 46 female, 38 unidentified $^{6}$. The mean age was 21.67, $(S D=0.99)$ years.

\section{Materials}

Helping and hurting were assessed through the THHT. Demographic, trait aggression $(M$ $=2.93, S D=0.79$, alpha $=0.90)$, and state hostility $(M=1.88, S D=0.53$, alpha $=0.93)$ scales from the previous studies were used.

Trait helpfulness. The 14-item helpfulness subscale of the Prosocial Battery Scale was used to assess trait helpfulness (Penner et al., 1995). Participants rated their agreement with statements (e.g., I have done volunteer work for a charity) on a 1 (Never) to 5 (Very Often) rating scale, $M=3.30, S D=0.58$, alpha $=0.79$.

Prime. Participants viewed either a Spirit-of-God image or an abstract image and wrote down any thoughts evoked by the image (Johnson, Li, Cohen, \& Okun, 2013; Study 3).

\section{Procedure}

After completing informed consent, participants were told that we were interested in understanding the relation between art preferences and puzzle performances. After receiving standard tangram task instructions, participants completed measures of trait aggression and trait

\footnotetext{
${ }^{5}$ In Studies 4 and 5, suspicion rate did not vary significantly by condition, $F<1.00, p>.20$

${ }^{6}$ Technical problems with Media Lab prevented some participants from completing the survey after the prime image was displayed; thus there were missing data on the demographic and state hostility questionnaires.
} 
helpfulness. Next, participants were randomly assigned to view and evaluate either an image portraying the Spirit-of-God or an abstract image. Following the prime, they chose 11 tangrams to assign to the other participant. Then, participants completed the post-experimental state hostility, motivation for tangram assignment, and demographic information. Finally, participants were probed for suspicion before being thanked and fully debriefed.

\section{Results}

\section{Preliminary Analyses}

Participants chose tangrams mostly from the medium category $\left(M_{\text {medium }}=4.50\right)$, followed by the easy $\left(M_{\text {easy }}=3.73\right)$, and hard categories $\left(M_{\text {hard }}=2.77\right)$. The mean helping, hurting and overall Tangram scores were $M \mathrm{~s}=2.76,1.87$, and 0.89 , respectively. The main effects and 2-way interactions of all pre-experimental measures with condition were tested in separate ANCOVAS for helping, hurting, and the difference score. Trait helpfulness yielded a significant interaction and was retained in the final model; other variables with non-significant effects were dropped.

\section{Main Analyses}

Overall Tangram score. A two-way ANOVA with prime (Spirit-Of-God/Neutral) and trait helpfulness revealed a significant effect of prime, $F(1,106)=5.42, p<.05, d=0.45$. Participants who saw the Spirit-Of-God image were more helpful/less hurtful those participants who saw the abstract image, $M \mathrm{~s}=1.54 ; 0.06,95 \%$ CIs [0.67, 2.42], [-0.85, 0.97], respectively.

In addition, there was a significant interaction between the prime and trait helpfulness, $F(1,106)=5.64, p<.05, \eta p^{2}=0.05$. Simple effects analyses revealed that trait helpfulness was significantly associated with a tendency to be more helpful/less hurtful in the Spirit-of-God condition, $F(1,55)=4.27, p<.05, b=0.97$, but not the neutral condition, $F<2.00, p>.10$. 
Adding state hostility as a covariate in this analysis did not change the effect of the prime, $F(1$, $\left.89^{7}\right)=5.00, p<.05, d=0.47$, suggesting that the effect of the prime on the tendency to help over hurt cannot be attributed to differences in state hostility.

Separate helping and hurting scores. The effect of the prime and trait helpfulness were tested in a mixed ANOVA with behavior as the within-subjects factor. The prime $\mathrm{X}$ behavior interaction was significant, $F(1,106)=6.32, p<.05, \eta p^{2}=0.06$ (Figure 1). Simple effects analyses revealed that Spirit-Of-God prime participants were more likely to help than neutral prime participants $(M \mathrm{~s}=3.14 \& 2.25,95 \%$ CIs [2.56, 3.72], [1.65, 2.85], respectively), $F(1,106)$ $=4.46, p<.05, d=0.41$. Conversely, neutral prime participants were significantly more likely to hurt Spirit-Of-God prime participants, $(M \mathrm{~s}=2.19 \& 1.60,95 \%$ CIs [1.79, 2.59], [1.21, 1.98], respectively), $F(1,106)=4.50, p<.05, d=0.41$.

Additionally, there was a three-way significant interaction between behavior type, prime, and trait helpfulness, $F(1,106)=5.64, p<.05, \eta p^{2}=0.05$. Follow up univariate analyses revealed that the two-way interaction between prime and trait helpfulness was significant for helpful, $F(1$, 106) $=4.72, p<.05, \eta p^{2}=0.04$, and hurtful behavior, $F(1,106)=4.56, p<.05, \eta p^{2}=0.04$. Simple effects analyses revealed that there was a positive and marginally significant effect of trait helpfulness on helping scores in the Spirit-of-God prime, $F(1,55)=3.44, p=.07, b=0.59$, but not the neutral prime, $F<2.00, p>.10$. Conversely, there was a negative and marginally significant effect of trait helpfulness on hurting scores in the Spirit-of-God prime, $F(1,55)=$ $3.46, p=.07, b=-0.38$, but not the neutral prime, $F<2.00, p>.10$.

To assess whether the prime effect resulted from state hostility, we covaried this variable in the above model. State hostility did not yield a significant main or interactive effect, $F s<3 ; p s$

\footnotetext{
${ }^{7}$ The smaller degrees of freedom result from missing values on state hostility, due to technical problems with Media Lab.
} 
$>0.05$. Importantly, the behavior $\mathrm{X}$ prime interaction remained significant, $F(1,89)=5.00, p<$ $.05, \eta p^{2}=0.05$, showing that the prime effect cannot be attributed to changes in state mood.

\section{Discussion}

In sum, a Spirit-Of-God prime manipulation increased helping behavior on the THHT using both scoring methods. Two-way interactions between trait helpfulness and the prime revealed that trait helpfulness significantly and positively predicted helping behavior on the THHT in the prosocial, but not neutral, contexts. This result contradicts the results obtained by previous studies in which trait helpfulness was significantly and positively associated with helpful behavior on the THHT (Saleem et al., 2015; Study 1). It is possible that the effect of trait helpfulness on helping within the THHT is weak in neutral contexts but enhanced within prosocial contexts. Surprisingly, trait aggression did not yield a significant main or interactive effect. Though primes can temporarily override the effects of trait tendencies, the fact that trait aggression was not significantly associated with the THHT in the neutral condition is puzzling and warrants attention in future research. Caution should be taken when interpreting these results given the small sample size of this study.

\section{STUDY 5: PROVOCATION EFFECT ON THE TANGRAM TASK AND EVALUATIONS}

There were two main goals for Study 5. First, we tested and compared the effects of provocation on tangram choices and on an established measure of aggression, i.e., negative evaluations of the other participant (e.g., Twenge et al., 2001). This experiment thus attempted to replicate the effects of provocation on tangram choices obtained in Saleem et al., (2015; Studies 5 and 6), while also adding a previously validated measure of aggression. This enabled us to compare these two aggression measures, a kind of sensitivity analysis. Second, we assessed and 
controlled for academic motivation, emotion regulation, and the big five personality traits to provide additional discriminant validity evidence.

\section{Method}

\section{Participants}

One hundred seventy participants from a large Midwestern University participated in this experiment for course credit. Fifteen were rated as suspicious based on debriefing questions, so their data were deleted. Of the remaining 168 participants, 37 were male, 131 female, 3 unidentified, Mage $=19.32$ years, $S D=2.49$.

\section{Materials}

Helping and hurting were assessed through the THHT. The physical aggression subscale of the Buss-Perry trait aggression scale $(M=3.25 ; S D=0.96$; alpha $=0.84)$ was included preexperimentally.

\section{Pre-experimental Measures}

Achievement Motivation: Participants rated their agreement with 10 statements (I am appealed by situations allowing me to test my abilities) on a 1 (Strongly Disagree) to 5 (Strongly Agree) scale (Lang \& Fries, 2006), $M=3.17 ; S D=0.46$, alpha $=.70$.

Emotional Regulation: Participants rated their agreement with 10 statements (I control my emotions by not expressing them) on a 1 (Strongly Disagree) to 5 (Strongly Agree) scale (Gross \& John, 2003). A 6-item reappraisal, $M=3.56, S D=0.59$, alpha $=.82$, and a 4-item suppression subscale were created, $M=2.69, S D=0.75$, alpha $=.77$.

Personality: Personality traits were assessed using the Ten-Item Personality Inventory (TIPI; Gosling, Rentfrow, \& Swann, 2003). The TIPI contains two items designed to assess each of the Big Five personality traits. The means and standard deviations for the extraversion, 
agreeableness, conscientiousness, emotional stability ${ }^{8}$, and openness to experience subscales were $M \mathrm{~s}=4.74,4.75,5.26,4.08,5.09 ; S D \mathrm{~s}=1.44,0.97,1.13,1.56,0.99$, alpha $=.72, .70, .61, .55$.

\section{Experimental-Manipulation}

Provocation. Provocation was induced through an essay task in which pairs of participants in adjacent cubicles are instructed to write an essay on abortion (Bushman \& Baumeister, 1998). They are told that they will evaluate the other participant's essay by providing written feedback. Participants in the provocation condition received negative feedback on all dimensions; those in the neutral condition received average feedback on all dimensions.

\section{Additional Outcome Measures}

Evaluation. Participants were told that the other participant had applied for a research assistant position in our lab (adapted from Twenge et al., 2001). They then rated the extent to which they thought the other participant is intelligent, skillful, competent, helpful, kind, warm, responsible, would do a good job, and should be hired, on 1 (Strongly Disagree) to 5 (Strongly Agree) scale. Finally, participants were told that research assistants get paid $\$ 7.25$ per hour in our lab and were asked to make a salary recommendation for the other participant on a continuous scale $(\$ 0-\$ 20.00), M=8.59, S D=3.51$.

\section{Procedure}

After consenting, participants were told that we are interested in understanding the relationship between writing style, impressions of others, and performance on a puzzle task. After receiving standard instructions on the tangram task through a video, participants completed pre-experimental questions. Next, participants completed the essay task by writing an essay either supporting or opposing abortion (their choice). Next, they were given the essay ostensibly

\footnotetext{
${ }^{8}$ Due to a programming error there was only one-item assessing emotional stability (I see myself as anxious, easily upset).
} 
written by the other participant and evaluated it. Then, they received the other participants' supposed evaluation of their essay which was designed to either provoke them or not. Next, they completed the tangram task and the evaluation of the other participant (counterbalanced order). Finally, participants were asked questions to assess suspicion, were thanked and fully debriefed.

\section{Results}

\section{Preliminary Analyses}

Participants chose tangrams mostly from the easy category $\left(M_{\text {easy }}=4.56\right)$, followed by the medium $\left(M_{\text {medium }}=3.26\right)$ and hard categories $\left(M_{\text {hard }}=3.18\right)$. For the pre-experimental and demographic variables, we tested their main effects and 2-way interactions with condition in separate ANOVAS for helping, hurting, and the difference score. Sex and the emotion suppression subscale yielded significant main effects; other variables did not yield any significant effects and thus were dropped from the main analyses.

\section{Main Analyses}

See Table 4 for descriptive statistics and zero-order correlations for outcome measures.

Overall Tangram score. A one-way (feedback: provocation or neutral) ANCOVA with the suppression subscale and sex as covariates revealed a significant effect of feedback, $F(1$, 148) $=9.22, p<.01, d=0.50$. Provoked participants were less helpful $/$ more hurtful compared to participants in the neutral condition, $M \mathrm{~s}=0.09 ; 2.52,95 \%$ CIs $[-1.05,1.22],[1.42,3.61]$, respectively. Additionally, suppression was positively associated with a tendency to help over hurt the other participant, $F(1,148)=7.55, p<.05, b=1.48$. Sex did not yield a significant effect.

Separate helping and hurting scores. A 2 (feedback: provocation or neutral) x 2 (behavior: helping or hurting) mixed ANCOVA was conducted with behavior as the within subjects factor and sex and suppression as covariates. The feedback $\mathrm{X}$ behavior interaction was 
significant, $F(1,148)=9.22, p<.01, \eta p^{2}=0.06$ (Figure 2 ). A simple effects analysis showed that provoked participants scored higher on hurting than participants in the neutral condition $(M \mathrm{~s}$ $=3.05 \& 1.68,95 \%$ CIs [2.46, 3.63], [1.12, 2.24], respectively), $F(1,148)=11.15, p<.01, d=$ 0.55. Conversely, participants in the neutral condition scored higher on helping than participants in the provocation condition, $(M \mathrm{~s}=4.19 \& 3.13,95 \%$ CIs $[3.57,4.82],[2.49,3.78]$, respectively), $F(1,148)=5.43, p<.05, d=0.38$.

In addition to the effect of condition, suppression yielded a significant two-way interaction with behavior type, $F(1,148)=7.55, p<.01, \eta p^{2}=0.05$. Simple effects analyses revealed that suppression was positively associated with helping, $F(1,148)=5.74, p<.05, b$ $=.74$, and negatively associated with hurting, $F(1,148)=7.30, p<.05, b=-0.75$. Sex did not yield a significant effect in these analyses.

Evaluation. A one-way (feedback: provocation or neutral) ANCOVA with suppression and sex as covariates revealed a significant effect of feedback, $F(1,148)=42.26, p<.001, d$ $=1.07, \eta p^{2}=0.22$. Participants in the neutral, relative to provoked, condition gave the other participant more positive evaluations, $M \mathrm{~s}=4.80 ; 3.58,95 \%$ CIs $[4.54,5.05]$, [3.32, 3.85], respectively. Participant sex and suppression were not significant in these analyses.

Salary. A one-way (feedback: provocation or neutral) ANCOVA with emotion regulation and sex as covariates revealed a significant effect of feedback, $F(1,148)=4.78, p<$ $.05, d=0.36, \eta p^{2}=0.03$. Participants in the neutral, relative to provoked, condition recommended a higher salary for the other participant, $M \mathrm{~s}=9.17 ; 7.93,95 \%$ CIs [8.39, 9.94], [7.12, 8.74], respectively. Participant sex and suppression were not significant in these analyses.

Comparison of hurting indices. To test if the experimental manipulation influenced the hurting scores from the tangram task differently than the salary recommendation for the other 
participant, a 2 (feedback: provocation/neutral) x 2 (outcome: hurting score from tangram task/monetary reward) mixed ANOVA was conducted with outcome as the within subjects factor. In these analyses we reverse-scored the salary measure so that higher scores indicate lower recommendations for salary. Additionally, both outcomes were standardized. As expected, the condition effect was significant, $F(1,150)=13.81, p<.01$. More importantly, none of the within-subject effects were significant, suggesting that the effect of provocation on aggressive behavior was not significantly different between these two laboratory measures of aggression.

\section{Discussion}

In sum, provocation increased hurting and decreased helping behavior as measured by the THHT. This effect was reliable for both scoring methods, the overall difference score and separate help and hurt scores, which are non-independent. The effect of provocation on hurting scores was similar in magnitude to the salary recommendation but smaller than the evaluations of the other participant. We suspect this may be because responses on the tangram task and the salary recommendation are representative of behavior whereas evaluation of the other participant represents an attitude, which is theoretically the mediating mechanism underlying the prime-tobehavior effect (see Wheeler \& DeMarree, 2009). Additionally, the provocation effect on tangram choices remained in Study 5 even after controlling for several personality variables, providing evidence for discriminant validity. Of the pre-experimental measures tested, only the suppression subscale of the emotion regulation scale yielded a significant main effect on THHT, such that suppression was related more to helping than hurting. Although there are few studies on the effect of trait emotional suppression on aggressive and prosocial behavior, previous studies show that participants who are instructed to suppress their emotions showed lower emotional reactions to visual stimuli than participants not instructed to suppress (Gross \& 
Levenson, 1997). Perhaps suppressing certain emotions leads to more prosocial behavior. Future research should explore this pattern more thoroughly.

\section{GENERAL DISCUSSION}

Using the Tangram Help/Hurt Task the present five studies tested correlational (Studies 1-3) and experimental (Studies 4-5) relationships between several variables theoretically and empirically related to aggressive and prosocial behavior. Studies 1-3 yielded convergent and discriminant validity evidence for the THHT using college-aged and adult samples and correlational designs. Across these three studies helpful and hurtful scores were significantly correlated with established trait assessments of aggression, forgiveness, empathy, perspective taking, state hostility, narcissism, moral reasoning, and importance for power and benevolence values. Meta-analytic results revealed that theoretically relevant individual difference measures of aggressive and prosocial behavior are significantly correlated with THHT performance in the expected directions. Results further revealed that THHT scores are not significantly associated with participant sex and social desirability concerns.

Experimental evidence from Study 4 validated the Tangram Task for use in studies priming prosocial behavior. Study 5 validated the Tangram Task for studies of provocation effects. Additional indices of aggression in Study 5 (evaluation of other participant and salary recommendations) significantly correlated with THHT scores. These effects remained while statistically controlling for other aggression-related personality and hostile mood indices. The effects of the experimental manipulation on the THHT were comparable and in some cases more sensitive than established indices of hurtful behavior (i.e., salary). Note that trait aggression does not significantly moderate the effects of the prime in the experimental studies. Furthermore, the correlation between trait aggression and scores on the THHT are smaller in experimental, than 
correlational studies. Both of these findings are consistent with the results of earlier studies (Saleem et al., 2015). Whether this implies a weak effect of trait aggression on the THHT or is due to the experimental prime attenuating a potential moderating effect of trait aggression is unclear at this point.

The intention/motivation assessments showed that assignment of harder puzzles was motivated by a desire to hurt (and not help) the other participant, whereas assignment of easier puzzles was motivated by a desire to help (and not hurt) the other participant. These consistent findings across multiple designs and methods provide further evidence of the key role played by intentions in both prosocial and antisocial domains (e.g., Graziano \& Habashi, 2010). Means from the present and previous research reveal that participants are more likely to help than hurt others in the THHT (Saleem et al., 2015).

\section{The Tangram Help/Hurt Task as a Valid Measure}

The evidence presented here coupled with the results of Saleem et al. (2015) convincingly demonstrate the utility of the THHT for assessing aggressive and prosocial behavior. This task has several advantages over established measures of helpful and hurtful behavior. First, the THHT allows simultaneous assessment of helping and hurting behavior. Although these two scores are negatively correlated, by assessing them in the same paradigm, researchers can use regression techniques to assess the unique and shared variances with correlational and experimental variables, and could even assess changes in helping/hurting choices over time to examine the roles of automatic and controlled processes (c.f., Graziano \& Habashi, 2010).

Second, researchers have the flexibility to evaluate what kind of scoring method is appropriate for their particular research design. For some studies, it may be appropriate to use an 
overall difference score that forces helping and hurting to be interdependent and on opposite sides of a continuum. For example, examining the extent to which participants choose helping over hurting (or vice versa) in a given context. For other studies, it may be more appropriate to use only help or hurt scores (e.g., Barlett \& Anderson, 2011; Saleem et al., 2015). This is especially useful when the predicted outcome is specific to helping or hurting behavior, but not necessarily both. For other researchers who are interested in helping and hurting behavior but are worried about the issue of interdependence and multicollinearity, it might make sense to use stronger adjustments to what constitutes as helping and hurting behavior. For example, counting only the number of hard tangram selections greater than 2 or 3 , and assigning zeros to participants who chose 0,1 , or 2 hard tangrams, would further reduce the correlation between the "help" and "hurt" scores and providence evidence for more extreme forms of aggression. If researchers are unclear as to which scoring method best answers their hypotheses, it might be best to report both scoring methods as done in the present and previous studies (Saleem et al., 2015). We caution researchers to be aware of the "researcher degrees of freedom" problem when choosing which scoring method to use (Simmons, Nelson, \& Simonsohn, 2011).

Third, the Tangram Task includes a medium category, allowing for a response that is neither aggressive nor prosocial (a common criticism levied against other validated aggression measures). Fourth, Tangrams can easily be used with adults and children. One can adjust the difficulty of the task for different populations by selecting a different set of Tangrams or by setting different time limits. Fifth, the cover story and various materials used are easily amenable to change, therefore allowing use in a diverse array of studies. Sixth, the THHT relies on simple count data compatible with paper or computer based administration, and does not require a complicated setup. Indeed, it is easily administered in online studies. Finally, because the 
Tangram Task inherently involves puzzle completion and assignment, task instructions should easily translate into other languages allowing this measure to be used cross-culturally.

\section{Limitations}

Several limitations of the THHT should be noted. First, all the studies reveal a strong negative correlation between the helping and hurting scores, when used separately. Individuals who score high on helpfulness by selecting a greater number of easy puzzles will score low on hurtfulness, and vice versa. Indeed, even after using our "greater than one" scoring procedure, the correlation between the helpful and hurtful scores remained high. This concern can addressed in several ways: (1) ignore the medium category for the analyses, thus reducing interdependence; (2) use the number of easy and difficult puzzles greater than one instead of raw scores so participants can obtain a score of 0 on both helpfulness and hurtfulness; (3) enter both helpful and hurtful scores as a within-subject factor in analyses; (4) use a difference score (helpful scorehurtful score); and (5) use regression procedures to examine the effects of an independent variable of either helping or hurting, while statistically controlling for the other Tangram score. Finally, one can further reduce the correlation between helpful and hurtful scores by setting more extreme rules for what counts as helpful or hurtful behavior.

A second limitation is that the correlations between relevant trait measures and the THHT are small to moderate. This is common throughout social psychology, especially when using college student samples that have restricted range, relative to other samples (e.g., Kalmore, 2015). For Mturk samples, the online setting may induce greater suspicion regarding the presence of another participant, thereby reducing the obtained correlations. Other issues surrounding Mturk samples include participants completing many similar studies and discussing studies on various different Mturk forums (Chandler, Mueller \& Paolacci, 2014). Two other 
possible contributors to the small magnitude of the correlations are: (a) the fact that general trait measures usually do not predict specific behavior very strongly; (b) the reported correlations did not adjust for unreliability of the trait measures, or of the THHT itself. In short, obtained correlations support the convergent validity predictions for this task, and are likely as good as convergent validity correlations for most other brief laboratory style measures of aggressive and prosocial behavior. Statistically, when selecting an appropriate measure, researchers should be aware of such effect sizes in order to select an appropriate sample size to have sufficient statistical power.

Future research also could compare the convergent validity of the THHT with additional aggressive and prosocial behavioral measures such as the competitive reaction time task (Bushman, 1995), prisoner's dilemma task (Rapoport, 1965), and intention to volunteer or donate to charities (Twenge et al., 2007). In addition, it is important to assess whether experimental manipulations of the accessibility of aggressive and prosocial cognitions influence choices on the THHT. Finally, the THHT should be used with different samples that have diverse demographic characteristics to better understand its generalizability.

\section{References}

Ames, D. R., Rose, P., \& Anderson, C. P. (2006). The NPI-16 as a short measure of narcissism. Journal of Research In Personality, 40, 440-450. doi:10.1016/j.jrp.2005.03.002. Anderson, C. A., \& Bushman, B. J. (2002). Human aggression. Annual Review of Psychology, 53, 27-51. doi:10.1146/annurev.psych.53.100901.135231.

Anderson, C. A., Deuser, W. E., \& DeNeve, K. M. (1995). Hot temperatures, hostile affect, hostile cognition, and arousal: Tests of a general model of affective aggression. Personality and Social Psychology Bulletin, 21, 434-448. doi:10.1177/0146167295215002. 
Anderson, C. A., Shibuya, A., Ihori, N., Swing, E. L., Bushman, B. J., Sakamoto, A., \& Saleem, M. (2010).Violent video game effects on aggression, empathy, and prosocial behavior in Eastern and Western countries: A meta-analytic review. Psychological Bulletin, 136, 151173. doi:10.1037/a0018251.

Barlett, C. P., \& Anderson, C. A. (2011). Reappraising the situation and its impact on aggressive behavior. Personality and Social Psychology Bulletin, 37, 1564-1573. doi:10.1177/0146167211423671.

Barlett, C. P., \& Anderson, C. A. (2013). Examining media effects: The General Aggression and General Learning Models. In E. Scharrer (Ed.), Media Effects/Media Psychology (pp. 120e). Blackwell-Wiley.

Batson, C. D. (2014). Are the 'principles in his nature, which interest him in the fortune of others,' moral?. In H. Putnam, S. Neiman, J. P. Schloss, H. Putnam, S. Neiman, J. P. Schloss (Eds.), Understanding moral sentiments: Darwinian perspectives (pp. 43-57). Piscataway, NJ, US: Transaction Publishers.

Berry, J. W., Worthington, E. J., O'Connor, L. E., \& Wade, N. G. (2005). Forgivingness, Vengeful Rumination, \& Affective Traits. Journal of Personality, 73,183-225. doi:10.1111/j.1467-6494.2004.00308.x.

Bonett, D. G. (2009). Meta-analytic interval estimation for bivariate correlations. Psychological Methods, 13,173-181. doi:10.1037/a0012868.

Bushman, B. J. (1995). Moderating role of trait aggressiveness in the effects of violent media on aggression. Journal of Personality and Social Psychology, 69, 950-960. doi:10.1037/00223514.69.5.950.

Bushman, B. J., \& Anderson, C. A. (1998). Methodology in the study of aggression: Integrating 
experimental and nonexperimental findings. In R. G. Geen, E. Donnerstein, R. G. Geen, E. Donnerstein (Eds.), Human aggression: Theories, research, and implications for social policy (pp. 23-48). San Diego, CA, US: Academic Press.

Bushman, B. J., \& Baumeister, R. F. (1998). Threatened egotism, narcissism, self-esteem, and direct and displaced aggression: Does self-love or self-hate lead to violence?. Journal of Personality and Social Psychology, 75, 219-229. doi:10.1037/0022-3514.75.1.219.

Buss, A. H., \& Perry, M. (1992). The Aggression Questionnaire. Journal of Personality and Social Psychology, 63, 452-459. doi:10.1037/0022-3514.63.3.452.

Chandler, J., Mueller, P., \& Paolacci, G. (2014). Nonnaïveté among Amazon Mechanical Turk workers: Consequences and solutions for behavioral researchers. Behavior research methods, 46, 112-130. doi:10.3758/s13428-013-0365-7.

Davis, M. H. (1983). Measuring individual differences in empathy: Evidence for a multidimensional approach. Journal of Personality and Social Psychology, 44, 113-126. doi:10.1037/0022-3514.44.1.113.

Davis, T. (2015). Group selection in the evolution of religion: Genetic evolution or cultural evolution?. Journal of Cognition and Culture, 15, 235-253. doi:10.1163/1568537312342149

DeWall, C. N., \& Anderson, C. A. (2011). The general aggression model. In P. R. Shaver, M. Mikulincer, P. R. Shaver, M. Mikulincer (Eds.) Human aggression and violence: Causes, manifestations, and consequences (pp. 15-33). Washington, DC, US: American Psychological Association.

DeWall, C. N., Anderson, C. A., \& Bushman, B. J. (2011). The general aggression model: 
Theoretical extensions to violence. Psychology of Violence, 1, 245-258. doi:10.1037/a0023842.

Dovidio, J. F., Piliavin, J. A., Schroeder, D. A., \& Penner, L. (2006). The social psychology of prosocial behavior. Mahwah, NJ, US: Lawrence Erlbaum Associates Publishers.

Gentile, D. A., Anderson, C. A., Yukawa, S., Ihori, N., Saleem, M., Ming, L. K., \& ... Sakamoto, A. (2009). The effects of prosocial video games on prosocial behaviors: International evidence from correlational, longitudinal, and experimental studies. Personality and Social Psychology Bulletin, 35, 752-763. doi:10.1177/0146167209333045.

Gilbert, F., Daffern, M., \& Anderson, C. A. (in press). The General Aggression Model and its application to violent offender assessment and treatment. In Peter Sturmey (Ed.), The Wiley Handbook of Violence and Aggression. Wiley-Blackwell.

Gosling, S. D., Rentfrow, P. J., \& Swann, W. J. (2003). A very brief measure of the Big-Five personality domains. Journal of Research In Personality, 37, 504-528. doi:10.1016/S00926566(03)00046-1.

Graziano, W. G., \& Habashi, M. M. (2010). Motivational processes underlying both prejudice and helping. Personality and Social Psychology Review, 14, 313-331. doi:10.1177/1088868310361239.

Gross, J. J., \& John, O. P. (2003). Individual differences in two emotion regulation processes: Implications for affect, relationships, and well-being. Journal of Personality and Social Psychology, 85, 348-362. doi:10.1037/0022-3514.85.2.348.

Gross, J. J. \& Levenson, R. W. (1997). Hiding feelings: The acute effects of inhibiting negative and positive emotion. Journal of Abnormal Psychology, 106, 95-103. doi:10.1037/0021843X.106.1.95. 
Hirsh, J. B., Galinsky, A. D., \& Zhong, C. (2011). Drunk, powerful, and in the dark: How general processes of disinhibition produce both prosocial and antisocial behavior. Perspectives On Psychological Science, 6, 415-427. doi:10.1177/1745691611416992.

Johnson, K. A., Li, Y. J., Cohen, A. B., \& Okun, M. A. (2013). Friends in high places: The influence of authoritarian and benevolent god-concepts on social attitudes and behaviors. Psychology of Religion and Spirituality, 5, 15-22. doi:10.1037/a0030138.

Joireman, J. A., Kuhlman, D. M., Van Lange, P. M., Doi, T., \& Shelley, G. P. (2003). Perceived rationality, morality, and power of social choice as a function of interdependence structure and social value orientation. European Journal of Social Psychology, 33, 413-437. doi:10.1002/ejsp.155.

Kalmoe, N. P. (2015). Trait aggression in two representative US surveys: Testing the generalizability of college samples. Aggressive behavior, 41, 171-188. doi:10.1002/ab.21547.

Krizan, Z. (2010). Synthesizer 1.0: A varying coefficient meta-analytic tool. Behavior Research Methods, 42, 863-870. doi:10.3758/BRM.42.3.863.

Lang, J. B., \& Fries, S. (2006). A revised 10-item version of the Achievement Motives Scale: Psychometric properties in german-speaking samples. European Journal of Psychological Assessment, 22, 216-224. doi:10.1027/1015-5759.22.3.216.

Liebert, R. M., \& Baron, R. A. (1972). Some immediate effects of televised violence on children's behavior. Developmental Psychology, 6, 469-475. doi:10.1037/h0032584.

Macrae, C. N., \& Johnston, L. (1998). Help, I need somebody: Automatic action and inaction. Social Cognition, 16, 400-417. doi:10.1521/soco.1998.16.4.400. 
Malle, B. F. (2011). Attribution theories: How people make sense of behavior. In D. Chadee, D. Chadee (Eds.), Theories in social psychology (pp. 72-95). Wiley-Blackwell.

Möller, I., \& Krahé, B. (2009). Exposure to violent video games and aggression in German adolescents: A longitudinal analysis. Aggressive Behavior, 35, 75-89. doi:10.1002/ab.20290.

Pashler, H., \& Wagenmakers, E. (2012). Editors' introduction to the special section on replicability in psychological science: A crisis of confidence?. Perspectives On Psychological Science, 7, 528-530. doi:10.1177/1745691612465253.

Penner, L. A., Fritzsche, B. A., Craiger, J. P., \& Freifeld, T. S. (1995). Measuring the prosocial personality. In J. N. Butcher, C. D. Spielberger, J. N. Butcher, C. D. Spielberger (Eds.), Advances in personality assessment, Vol. 10 (pp. 147-163). Hillsdale, NJ, England: Lawrence Erlbaum Associates, Inc.

Piff, P. K., Kraus, M. W., Côté, S., Cheng, B. H., \& Keltner, D. (2010). Having less, giving more: The influence of social class on prosocial behavior. Journal of Personality and Social Psychology, 99, 771-784. doi:10.1037/a0020092.

Prot, S., Anderson, C. A., Gentile, D. A., Warburton, W., Saleem, M., Groves, C. L., \& Brown, S. C. (2015). Media as agents of socialization. In J. E. Grusec, P. D. Hastings, J. E. Grusec, P. D. Hastings (Eds.), Handbook of socialization: Theory and research (2nd ed.) (pp. 276300). New York, NY, US: Guilford Press.

Rapoport, A., \& Chammah, A. M. (1965). Sex differences in factors contributing to the level of cooperation in the Prisoner's Dilemma game. Journal of Personality and Social Psychology, 2, 831-838. doi:10.1037/h0022678. 
Reynolds, W. M. (1982). Development of reliable and valid short forms of the Marlowe-Crowne Social Desirability Scale. Journal of Clinical Psychology, 38, 119-125. doi: 10.1002/10974679(198201)38:1<119::AID-JCLP2270380118>3.0.CO;2-I.

Saleem, M., Anderson, C. A., \& Barlett, C. P. (2015). Assessing helping and hurting behaviors through the Tangram Help/Hurt Task. Personality and Social Psychology Bulletin, 41, 1345-1362. doi:10.1177/0146167215594348.

Saleem, M., Prot, S., Cikara, M., Lam, B. P., Anderson, C. A., \& Jelic, M. (2015). Cutting Gordian knots: Reducing prejudice through attachment security. Personality and Social Psychology Bulletin, 41, 1560-1574. doi:10.1177/0146167215601829.

Schwartz, S. H. (2010). Basic values: How they motivate and inhibit prosocial behavior. In M.

Mikulincer, P. R. Shaver, M. Mikulincer, P. R. Shaver (Eds.), Prosocial motives, emotions, and behavior: The better angels of our nature (pp. 221-241). Washington, DC, US: American Psychological Association.

Simmons, J. P., Nelson, L. D., \& Simonsohn, U. (2011). False-positive psychology undisclosed flexibility in data collection and analysis allows presenting anything as significant. Psychological science, 22, 1359-1366. doi:10.1177/0956797611417632.

Spellman, B. A. (2013). Introduction to the special section on advancing science. Perspectives On Psychological Science, 8, 412-413. doi:10.1177/1745691613493245.

Tremblay, P. F., \& Ewart, L. A. (2005). The Buss and Perry Aggression Questionnaire and its relations to values, the Big Five, provoking hypothetical situations, alcohol consumption patterns, and alcohol expectancies. Personality and Individual Differences, 38, 337-346. doi:10.1016/j.paid.2004.04.012.

Twenge, J. M., Baumeister, R. F., Tice, D. M., \& Stucke, T. S. (2001). If you can't join them, 
beat them: Effects of social exclusion on aggressive behavior. Journal of Personality and Social Psychology, 81, 1058-1069. doi:10.1037/0022-3514.81.6.1058.

Twenge, J. M., Baumeister, R. F., DeWall, C. N., Ciarocco, N. J., \& Bartels, J. M. (2007). Social exclusion decreases prosocial behavior. Journal of Personality and Social Psychology, 92, 56-66. doi:10.1037/0022-3514.92.1.56.

van Baaren, R. B., Holland, R. W., Kawakami, K., \& van Knippenberg, A. (2004). Mimicry and Prosocial Behavior. Psychological Science, 15, 71-74. doi:10.1111/j.09637214.2004.01501012.x.

Warburton, W., \& Anderson, C. A. (in press). On the clinical applications of the General Aggression Model to understanding domestic violence. In R. A. Javier \& W. G. Herron (Eds.), Understanding Domestic Violence: Theories, Challenges, Remedies. Rowman \& Littlefield Publishers, Inc.

Webster, G. D., DeWall, C. N., Pond, R. J., Deckman, T., Jonason, P. K., Le, B. M., \& ... Bator, R. J. (2014). The brief aggression questionnaire: Psychometric and behavioral evidence for an efficient measure of trait aggression. Aggressive Behavior, 40, 120-139. doi:10.1002/ab.21507.

Wheeler, S. C., \& DeMarree, K. G. (2009). Multiple mechanisms of prime-to-behavior effects. Social And Personality Psychology Compass, 3, 566-581. doi:10.1111/j.17519004.2009.00187.x.

Tables

Table 1: Means, standard deviations, and alphas for all measures used in Studies 1-3 


\begin{tabular}{|c|c|c|c|c|c|c|c|c|}
\hline \multirow[b]{2}{*}{ Measures } & \multicolumn{3}{|c|}{ Study $1 \mathrm{~N}=233$} & \multicolumn{3}{|c|}{ Study $2 \mathrm{~N}=241$} & \multicolumn{2}{|c|}{ Study $3 \mathrm{~N}=230$} \\
\hline & Mean & SD & alpha & Mean & SD & Alpha & Mean & $\mathrm{SD}$ \\
\hline Easy & 4.45 & 2.66 & & 5.85 & 3.39 & & 6.04 & 3.08 \\
\hline Medium & 4.00 & 1.63 & & 2.93 & 1.81 & & 3.14 & 1.77 \\
\hline Hard & 2.55 & 1.84 & & 2.22 & 2.54 & & 1.82 & 1.93 \\
\hline Forgiveness & 3.43 & 0.75 & 0.80 & & & & & \\
\hline State Hostility & 1.91 & 0.60 & 0.94 & & & & & \\
\hline Power & 5.31 & 1.34 & 0.71 & & & & & \\
\hline Benevolence & 7.11 & 1.21 & 0.51 & & & & & \\
\hline BP Physical & 2.69 & 1.06 & 0.80 & 2.77 & 1.58 & 0.78 & & \\
\hline BP Verbal & 3.57 & 1.14 & 0.71 & 3.69 & 1.40 & 0.70 & & \\
\hline BP Anger & 2.61 & 1.00 & 0.78 & 2.54 & 1.41 & 0.82 & & \\
\hline BP Hostility & 2.86 & 1.09 & 0.80 & 3.27 & 1.54 & 0.76 & & \\
\hline BP Composite & 2.87 & 0.82 & 0.89 & 3.07 & 1.13 & 0.86 & & \\
\hline Empathy & & & & 3.93 & 0.85 & 0.90 & & \\
\hline Perspective Taking & & & & 3.77 & 0.76 & 0.86 & & \\
\hline Narcissism & & & & 0.25 & 0.23 & 0.84 & & \\
\hline Social Desirability & & & & 0.44 & 0.26 & 0.79 & 0.46 & 0.27 \\
\hline $\begin{array}{l}\text { Social } \\
\text { Responsibility }\end{array}$ & & & & & & & 3.35 & 0.48 \\
\hline Moral Reasoning & & & & $\infty$ & & & 3.82 & 0.65 \\
\hline Motivation to help & 3.48 & 2.61 & - & 3.73 & 1.19 & 0.90 & 3.80 & 1.11 \\
\hline Motivation to hurt & 1.72 & 1.63 & 0.75 & 2.16 & 1.21 & 0.90 & 2.08 & 1.09 \\
\hline
\end{tabular}

Table 2. Zero-order correlations of tangram scores with other measures used in Studies 1-3

\begin{tabular}{|l|l|l|l|l|l|l|l|l|}
\hline & \multicolumn{3}{|l|}{ Study 1 N 233} & \multicolumn{2}{l|}{ Study 2 N 241} & \multicolumn{2}{l|}{ Study 3 N = 230} \\
\hline Zero-order $r \mathbf{s}$ & Helping & Hurting & $\begin{array}{l}\text { Help- } \\
\text { Hurt }\end{array}$ & Helping & Hurting & $\begin{array}{l}\text { Help- } \\
\text { Hurt }\end{array}$ & Helping & Hurting \\
\hline Hurting & $-0.73^{* * *}$ & & & $-0.76^{* * *}$ & & & $-0.74^{* * *}$ & \\
\hline Help-Hurt & $0.96^{* * *}$ & $-0.89^{* * *}$ & & $0.96^{* * *}$ & $-0.91^{* * *}$ & & $0.97^{* * *}$ & $-0.88^{* * *}$ \\
\hline Forgiveness & $0.24^{* *}$ & $-0.24^{* *}$ & $0.25^{* * *}$ & & & & & \\
\hline State Hostility & -0.10 & $0.15^{*}$ & $-0.13+$ & & & & & \\
\hline Benevolence & $0.14 *$ & -0.03 & 0.11 & & & & & \\
\hline Power & -0.09 & $0.22^{* *}$ & $-0.15^{*}$ & & & & & \\
\hline BP Physical & -0.07 & $0.16^{*}$ & -0.11 & $-0.19^{* *}$ & $0.17^{*}$ & $-0.19^{* *}$ & & \\
\hline BP Verbal & 0.03 & 0.01 & 0.01 & -0.12 & 0.10 & -0.12 & & \\
\hline BP Anger & -0.06 & $0.16^{*}$ & -0.11 & $-0.13+$ & $0.17^{*}$ & -0.10 & & \\
\hline BP Hostility & -0.11 & $0.18^{*}$ & $-0.14^{*}$ & $-0.20^{* *}$ & $0.17^{*}$ & $-0.19^{* *}$ & & \\
\hline BP Composite & -0.08 & $0.18^{*}$ & $-0.13+$ & $-0.21^{* *}$ & $0.17^{*}$ & $-0.20^{* *}$ & & \\
\hline Empathy & & & & $0.23^{* *}$ & $-0.19^{* *}$ & $0.22^{* *}$ & & \\
\hline Perspective Taking & & & & $0.19^{* *}$ & $-0.15^{*}$ & $0.17^{*}$ & & \\
\hline
\end{tabular}




\begin{tabular}{|l|l|l|l|l|l|l|l|l|}
\hline Narcissism & & & & $-0.13+$ & $0.19 * *$ & $-0.16^{*}$ & & \\
\hline Social Desirability & & & & -0.02 & 0.01 & -0.02 & -0.04 & -0.01 \\
\hline $\begin{array}{l}\text { Social } \\
\text { Responsibility }\end{array}$ & & & & & & & $0.13+$ & $-0.13+$ \\
\hline Moral Reasoning & & & & & & & $0.18^{*}$ & $-0.20^{* *}$ \\
\hline Motivation to help & $0.67 * * *$ & $-0.60 * * *$ & $0.69 * * *$ & $0.82^{* * *}$ & $-0.80^{* * *}$ & $0.84^{* * *}$ & $0.71^{* * *}$ & $-0.69^{* * *}$ \\
\hline Motivation to hurt & $-0.59 * * *$ & $0.57 * * *$ & $-0.63 * * *$ & $-0.77^{* * *}$ & $0.82^{* * *}$ & $-0.83^{* * *}$ & $-0.72^{* * *}$ & $0.66^{* * *}$ \\
\hline Sex^ & 0.07 & -0.04 & 0.06 & 0.04 & -0.10 & 0.06 & 0.03 & -0.01 \\
\hline Means & 3.48 & 1.72 & 1.76 & 4.91 & 1.59 & 3.32 & 5.07 & 1.17 \\
\hline $\begin{array}{l}\text { Standard } \\
\text { Deviations }\end{array}$ & 2.61 & 1.63 & 3.96 & 3.30 & 2.24 & 5.21 & 3.02 & 1.63 \\
\hline
\end{tabular}

${ }^{\wedge} \operatorname{Sex}(0=$ female; $1=$ male $),+p=.05, * p<.05, * * p<.01, * * * p<.001$.

Table 3. Meta-analysis of aggregate Pearson correlations between individual difference variables and helping and hurting scores on the Tangram Help/Hurt Task derived from Studies 1-3 of the present paper and past research (Saleem, Anderson, \& Barlett, 2015: Studies 1-3).

\begin{tabular}{|c|c|c|c|c|c|c|c|c|c|c|c|c|}
\hline & \multicolumn{5}{|c|}{ Helping } & \multicolumn{5}{|c|}{ Hurting } & \multirow[b]{2}{*}{$\begin{array}{c}\text { Lower } \\
95 \%\end{array}$} & \multirow[b]{2}{*}{$\begin{array}{c}\text { Help- } \\
\text { Hurt- } \\
\text { r+ }\end{array}$} \\
\hline & $\begin{array}{c}\text { Lower } \\
95 \%\end{array}$ & $\begin{array}{c}\text { Help- } \\
\text { r+ }\end{array}$ & $\begin{array}{c}\text { Upper } \\
95 \%\end{array}$ & $\mathrm{~K}$ & $\mathrm{~N}$ & $\begin{array}{c}\text { Lower } \\
95 \%\end{array}$ & $\begin{array}{c}\text { Hurt- } \\
\mathrm{r}+\end{array}$ & $\begin{array}{c}\text { Upper } \\
95 \%\end{array}$ & $\mathrm{~K}$ & $\mathrm{~N}$ & & \\
\hline BP-Anger & -0.23 & -0.17 & -0.10 & 4 & 813 & 0.16 & 0.23 & 0.29 & 4 & 813 & -0.25 & -0.19 \\
\hline BP-Hostility & -0.24 & -0.17 & -0.10 & 4 & 813 & 0.12 & 0.19 & 0.25 & 4 & 813 & -0.25 & -0.18 \\
\hline BP-Physical & -0.22 & -0.16 & -0.09 & 4 & 813 & 0.13 & 0.20 & 0.27 & 4 & 813 & -0.25 & -0.19 \\
\hline BP-Verbal & -0.15 & -0.09 & -0.02 & 4 & 813 & 0.02 & 0.09 & 0.16 & 4 & 813 & -0.16 & -0.09 \\
\hline BP-Total & -0.26 & -0.20 & -0.13 & 4 & 813 & 0.16 & 0.23 & 0.29 & 4 & 813 & -0.29 & -0.22 \\
\hline Empathy & 0.10 & 0.20 & 0.29 & 2 & 418 & -0.30 & -0.21 & -0.11 & 2 & 418 & 0.11 & 0.21 \\
\hline Motivation to help & 0.72 & 0.75 & 0.77 & 6 & 1189 & -0.73 & -0.70 & -0.67 & 6 & 1189 & 0.75 & 0.77 \\
\hline Motivation to hurt & -0.70 & -0.67 & -0.63 & 6 & 1189 & 0.62 & 0.66 & 0.69 & 6 & 1189 & -0.74 & -0.71 \\
\hline Narcissism & -0.29 & -0.20 & -0.09 & 2 & 387 & 0.16 & 0.26 & 0.35 & 2 & 387 & -0.32 & -0.23 \\
\hline Perspective taking & 0.12 & 0.22 & 0.31 & 2 & 418 & -0.29 & -0.20 & -0.11 & 2 & 418 & 0.12 & 0.22 \\
\hline Prosocialness & 0.11 & 0.21 & 0.31 & 2 & 339 & -0.20 & -0.10 & 0.01 & 2 & 339 & 0.06 & 0.17 \\
\hline Participant sex & -0.03 & 0.03 & 0.09 & 6 & 1189 & -0.09 & -0.03 & 0.03 & 6 & 1189 & -0.03 & 0.03 \\
\hline State hostility & -0.22 & -0.12 & -0.02 & 2 & 410 & 0.06 & 0.16 & 0.25 & 2 & 410 & -0.24 & -0.15 \\
\hline Social desirability & -0.06 & 0.02 & 0.10 & 3 & 617 & -0.09 & -0.01 & 0.07 & 3 & 617 & -0.07 & 0.01 \\
\hline
\end{tabular}

Note: All listed independent variables significantly predicted all three tangram scores at $p<.05$ except for participant sex and social desirability. BP = Buss-Perry, Lower and Upper 95\% refers to confidence intervals, $K=$ number of studies, $N=$ sum of the sample sizes of the studies included in the analysis.

Table 4. Zero-order correlations of outcome measures in Study 5. 


\begin{tabular}{|l|l|l|l|l|l|}
\hline & $\begin{array}{l}\text { Tangram } \\
\text { overall score }\end{array}$ & $\begin{array}{l}\text { Tangram } \\
\text { Help }\end{array}$ & $\begin{array}{l}\text { Tangram } \\
\text { Hurt }\end{array}$ & Salary & Evaluation \\
\hline $\begin{array}{l}\text { Tangram } \\
\text { overall score }\end{array}$ & & & & & \\
\hline Tangram Help & $.94^{* * *}$ & & & & \\
\hline Tangram Hurt & $-.93^{* * *}$ & $-.74^{* * *}$ & & & \\
\hline Salary & $.23^{*}$ & $.20^{*}$ & $-.22^{*}$ & & \\
\hline Evaluation & $.27^{* *}$ & $.20^{*}$ & $-.31^{* * *}$ & $.36^{* * *}$ & \\
\hline Means & 1.34 & 3.67 & 2.33 & 8.59 & 4.21 \\
\hline $\begin{array}{l}\text { Standard } \\
\text { Deviations }\end{array}$ & 5.22 & 2.91 & 2.68 & 3.51 & 1.29 \\
\hline
\end{tabular}

Ns $=152-153, * p<.05, * * p<.01, * * * p<.001$.

Figures

Figure 1. Helpful and Hurtful Behavior as a Function of Prime (Spirit-of-God or Neutral)

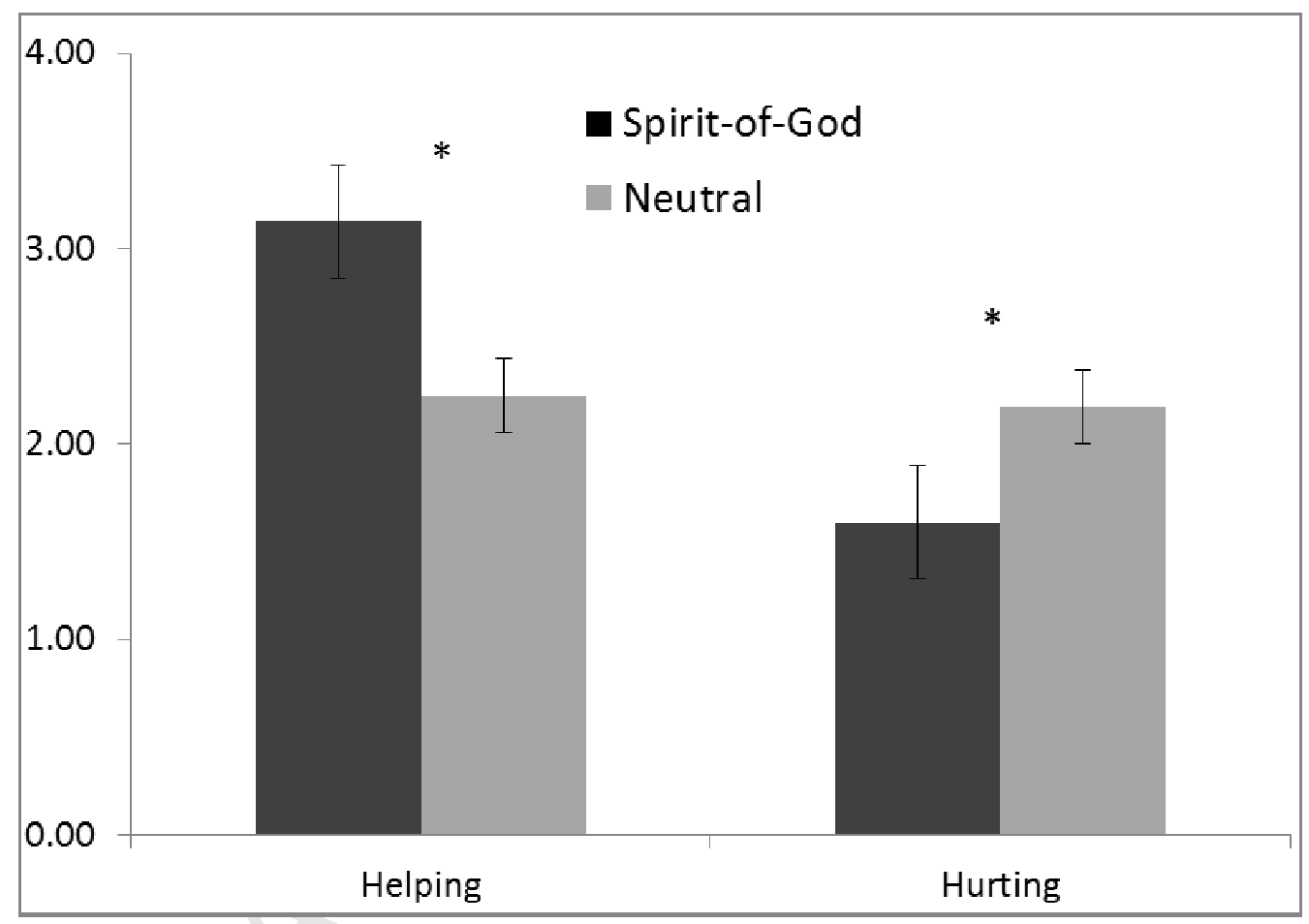

Note. Bars represent standard errors, $* p<0.5$

Figure 2. Helpful and Hurtful Behavior as a Function of Prime (Provoke or Neutral) 


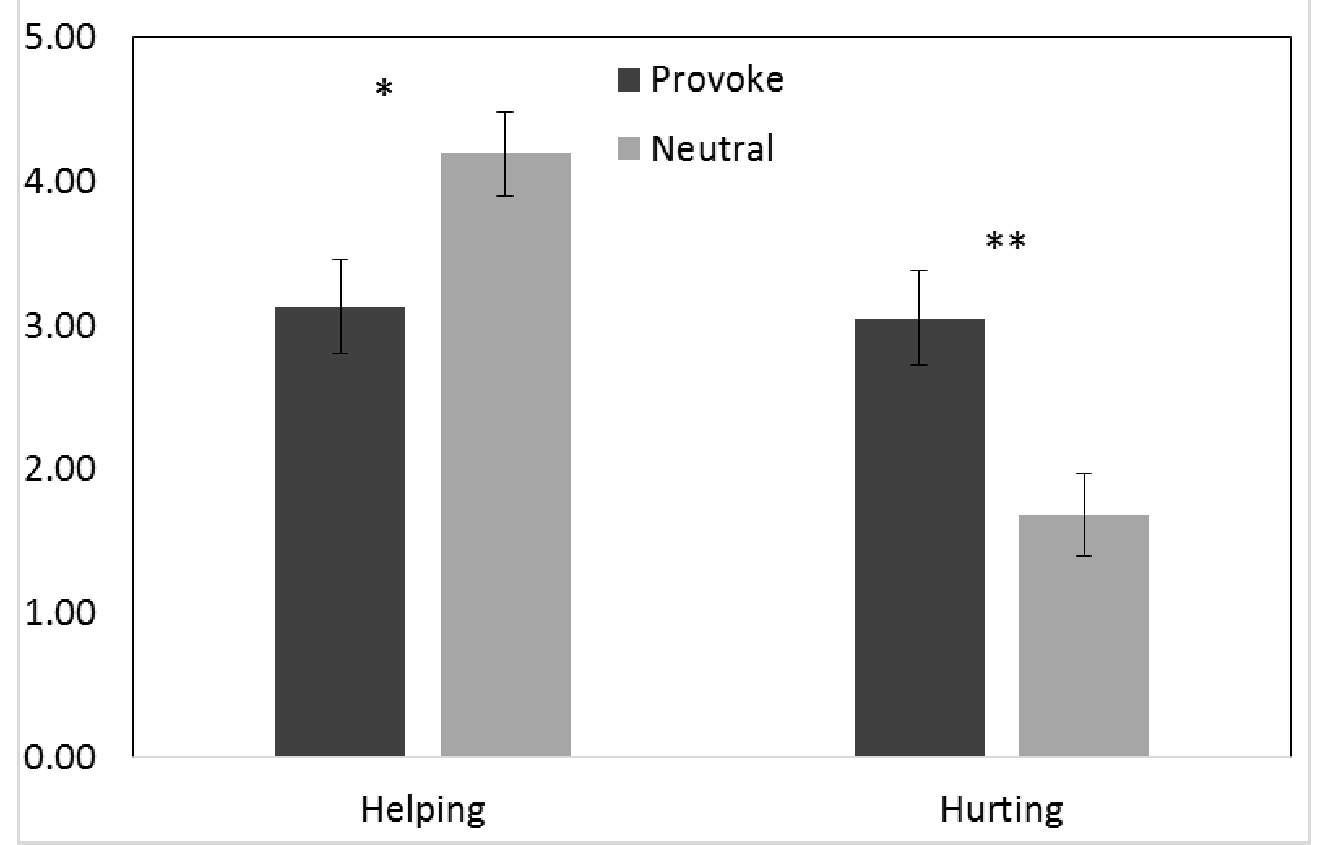

Note. Bars represent standard errors, ${ }^{* *} p<0.01,{ }^{*} p<.05$ 